\title{
Magnetic Force Driven Nanogenerators as a Noncontact Energy Harvester and Sensor
}

\author{
Nuanyang Cui, ${ }^{\dagger}$ Weiwei Wu, ${ }^{\dagger}$ Yong Zhao, ${ }^{\ddagger}$ Suo Bai, ${ }^{\dagger}$ Leixin Meng, ${ }^{\dagger}$ Yong Qin, ${ }^{* \dagger}$ and Zhong Lin Wang ${ }^{*}, \S$ \\ ${ }^{\dagger}$ Institute of Nanoscience and Nanotechnology, Lanzhou University, Lanzhou 730000, China \\ ${ }^{\ddagger}$ Beijing National Laboratory for Molecular Sciences (BNLMS), Institute of Chemistry, Chinese Academy of Sciences, Beijing \\ 100190, China \\ ${ }^{\S}$ School of Materials Science and Engineering, Georgia Institute of Technology, Atlanta, Georgia 30332-0245, United States
}

\section{Supporting Information}

ABSTRACT: Nanogenerator has been a very important energy harvesting technology through directly deforming piezoelectric material. Here, we report a new magnetic force driven contactless nanogenerator (CLNG), which avoids the direct contact between nanogenerator and mechanical movement source. The CLNG can harvest the mechanical movement energy in a noncontact mode to generate electricity. Their output voltage and current can be as large as $3.2 \mathrm{~V}$ and $50 \mathrm{nA}$, respectively, which is large enough to power up a liquid crystal display. We also demonstrate a means by which a magnetic sensor can be built.

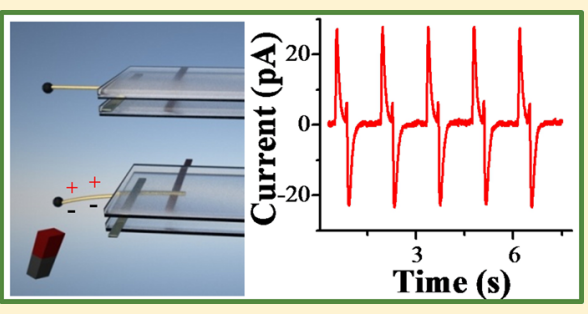

KEYWORDS: Nanogenerator, ZnO micro/nanowires, PZT nanowires, energy harvesting, noncontact mode

$\mathrm{I}_{\mathrm{s}}^{\mathrm{n}}$ $n$ recent years, energy-harvesting technologies that can scavenge every kinds of energy from our living environment to power micro/nanodevices have attracted increasing massive attention. $^{1-7}$ Because of the ability of generating electricity from all sorts of mechanical motions, piezoelectric nanogenerator plays a vital role for self-powered devices/ systems. $^{8-10}$ The key material of nanogenerators is the piezoelectric semiconductor and piezoelectric ferroelectric, which can create piezoelectric field under deformation. Electrons in the external circuit are driven to flow back and forth by this piezoelectric field. In this way, nanogenerators convert the energy of mechanical motion into electricity. ${ }^{11}$ The first nanogenerator is demonstrated by pushing the $\mathrm{ZnO}$ nanowire array with an atomic force microscopy (AFM) tip. ${ }^{12}$ After that, dc nanogenerator, ${ }^{13}$ ac nanogenerator, ${ }^{11}$ wearable fiber nanogenerator, ${ }^{14}$ and integrated high output nanogenerators $^{10,15-17}$ were invented. For all of these nanogenerators (NGs), their driven modes can be classified into the following categories: friction between two substrates, ${ }^{14}$ bending of a flexible substrate, ${ }^{17}$ pressing of flexible polymer, ${ }^{16}$ and ultrasonic wave driving mode. ${ }^{18}$ These driving modes can be called as a contact mode, which has a common characteristic of the direct contact between the mechanical movement source and the piezoelectric material. In some particular situations under which the mechanical movements are not suitable to generate electricity, such as in vivo circumstances, self-powered devices need to be charged in a noncontact mode. As a result, it is helpful and necessary to explore a new kind of contactless nanogenerator (CLNG) to power the functional devices without contacting with them. In this paper, we present two kinds of magnetic force driven CLNG composed of a single $\mathrm{ZnO}$ microwire and lead zirconate titanate (PZT) nanowires, respectively. By combining micro/nanowire with magnetic material, these nanogenerators show the ability of harvesting energy from the varying magnetic field. The maximum output voltage and current of CLNGs have reached $3.2 \mathrm{~V}$ and $50 \mathrm{nA}$. Also, a CLNG is used to successfully lighten a liquid crystal display (LCD) screen.

Contactless Nanogenerator Made with a Single $\mathrm{ZnO}$ Wire (SCLNG). The basic structure of SCLNG is two glass plates sandwiching a $\mathrm{ZnO}$ microwire as shown in Figure 1a. It is fabricated through the following steps. First, photolithography and magnetic sputtering are used to deposit a strip of silver (Ag) electrode with a width of $50 \mu \mathrm{m}$ on a piece of glass plate. After that, a $\mathrm{ZnO}$ microwire (about $500 \mu \mathrm{m}$ in length) is placed on this glass plate keeping its length direction perpendicular to the plate's edge. Then, a photoresist layer is spin-coated on the top of the $\mathrm{ZnO}$ microwire. Subsequently, only a $50 \mu \mathrm{m}$ wide photoresist strip parallel to the plate's edge is remained using photolithography technology. After a baking process on the hot plate at $150{ }^{\circ} \mathrm{C}$ for $30 \mathrm{~min}$, the photoresist strip is robust enough to fix the $\mathrm{ZnO}$ microwire onto the glass plate. Then another glass plate covered with a $50 \mu \mathrm{m}$ wide palladium $(\mathrm{Pd})$ striplike electrode on its edge is put on the top of the $\mathrm{ZnO}$ microwire. These two pieces of glass and $\mathrm{ZnO}$ microwire in the middle form a sandwich structure. The structure is similar to the force sensor. ${ }^{19}$ The $\mathrm{ZnO}$ microwire and Pd electrode form a Schottky contact. Finally, the $\mathrm{ZnO}$ microwire's top end is dipped into a mixed solution of $\mathrm{Fe}_{3} \mathrm{O}_{4}$ fine powder and paraffin. After taking it out from the solution and cooling down, the

Received: April 20, 2012

Revised: June 2, 2012 

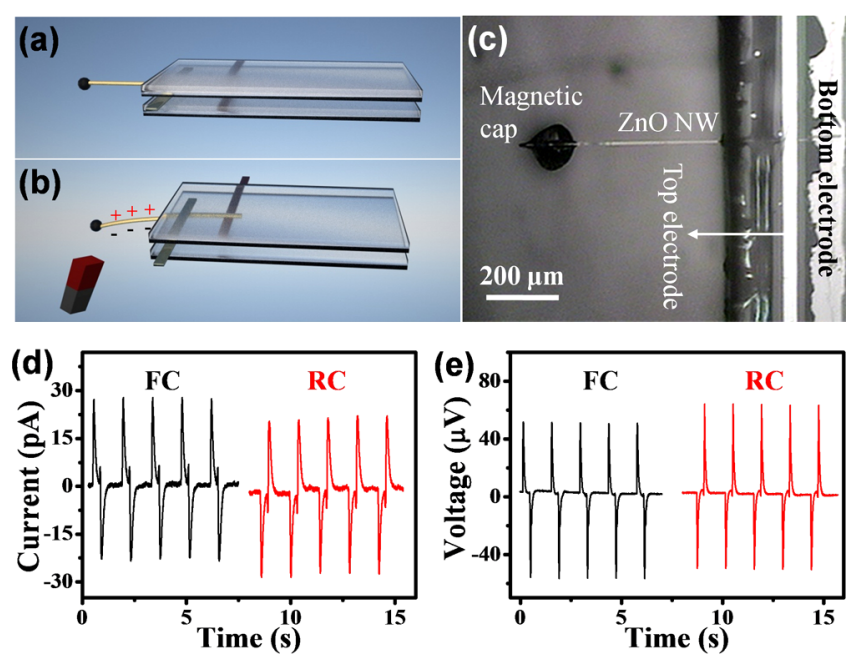

Figure 1. Structure and performance of the SCLNG. (a) Schematic of the SCLNG. A ZnO nanowire with magnetic cap is sandwiched by two electrodes. (b) Working mechanism of the SCLNG. When $\mathrm{ZnO}$ nanowire is bended, piezoelectric potential between two sides of $\mathrm{ZnO}$ nanowire drives electrons flowing and thus generates electricity. (c) A photograph of the SCLNG. (d,e) The output current and voltage of the SCLNG, despectively. The black curves represent the output signals under FC, and the red curves are the output signals under RC.

$\mathrm{ZnO}$ microwire's top is covered with a magnetic composite cap. Figure 1c shows the optical graph of a SCLNG.

The work mechanism of SCLNG is shown in Figure 1b. As the bar magnet approaches the magnetic cap on the top of $\mathrm{ZnO}$ microwire from the distance, the cap induces a magnetic force because of the variation of the magnetic field, which leads to the bending of $\mathrm{ZnO}$ microwire. As a result, there is a strain in the microwire, and piezopotential appears on the surface of the microwire. The stretching side of the microwire has positive potential, and the compressed side has negative potential. The electrons in the external circuit will flow through loads from low potential side to high potential side and accumulate at the interface between the $\mathrm{ZnO}$ microwire and circuit because of the Schottky barrier. ${ }^{20}$ When the bar magnet leaves from the magnetic top of the $\mathrm{ZnO}$ microwire, the strain and piezopotential disappear, and the accumulated electrons will flow back in external circuit. If the bar magnet approaches and leaves the $\mathrm{ZnO}$ microwire periodically, electrons will flow back and forth in external circuit. During these processes, SCLNG converts the mechanical energy that drives the back and forth movement of the bar magnet into electricity without a direct contact of the $\mathrm{ZnO}$ microwire and mechanical motion source (bar magnet).

The electrical measurements are carried out in a Farady cage to shield external electromagnetic noises. The bar magnet fixed onto a linear motor approaches and leaves the magnetic cap at a given driving frequency to supply mechanical energy. To avoid the influence of electromagnetic induction, the two conductive wires are twisted with each other. The voltage signal and current signal were measured through Stanford Research Systems (low-noise preamplifier SR560 and low-noise current preamplifier SR570). The input resistances of the preamplifiers were $100 \mathrm{M} \Omega$ and $10 \mathrm{k} \Omega$, respectively. Figure $1 \mathrm{~d}$,e shows the output current and voltage of the SCLNG, respectively. FC means forward connection, that is, the positive probe of the measurement system connecting with SCLNG's positive end and the negative probe connecting with the negative end. RC means reversed connection. For forward connection, the measured maximum output voltage is $60 \mu \mathrm{V}$, and maximum output current is around $30 \mathrm{pA}$ at the driving frequency of 0.7 $\mathrm{Hz}$. These outputs are generated at a maximum strain of $0.35 \%$ on stretched/compressed surface of $\mathrm{ZnO}$ microwire. The corresponding straining rate is about $1.75 \% \cdot \mathrm{s}^{-1}$. When the measurement system is reversely connected to the SCLNG, the output signals reversed too. So the output signals satisfy the switching-polarity test. ${ }^{21}$ In addition, when the magnetic cap on the top of $\mathrm{ZnO}$ microwire is cut off and $\mathrm{ZnO}$ microwire cannot be bent, the output signal disappears, which rules out the possibility that the signal is coming from the electromagnetic induction. Furthermore, the linear superposition test is carried out to prove the correctness of output signals. SCLNG A with $30.5 \mu \mathrm{V}$ output voltage and $11.55 \mathrm{pA}$ output current is connected with SCLNG B (output voltage of $54 \mu \mathrm{V}$ and output current of $25.35 \mathrm{pA}$ ) in series and in parallel at different configurations. The measurement results are shown in Supporting Information Figure $S$ and Table I. The super-

Table I. Summary of Current and Voltage Output When the SCLNG A and SCLNG B Are Connected in Various Configurations

\begin{tabular}{lccccc} 
& \multicolumn{2}{c}{ current } & & \multicolumn{2}{c}{ voltage } \\
\cline { 2 - 3 } \cline { 5 - 6 } MWG & $\begin{array}{c}\text { forward } \\
\text { connecting } \\
(\mathrm{pA})\end{array}$ & $\begin{array}{c}\text { reverse } \\
\text { connection } \\
(\mathrm{pA})\end{array}$ & & $\begin{array}{c}\text { forward } \\
\text { connecting } \\
(\mu \mathrm{V})\end{array}$ & $\begin{array}{c}\text { reverse } \\
\text { connection } \\
(\mu \mathrm{V})\end{array}$ \\
$\mathrm{A}$ & 11.55 & -11.73 & & 30.5 & -33.7 \\
$\mathrm{~B}$ & 25.35 & -24.35 & & 54 & -57.3 \\
$\mathrm{~A}+\mathrm{B}$ & 36.27 & -35.78 & & 85.63 & -92.1 \\
A-B & -12.55 & 13.25 & & -20.2 & 19.65 \\
\hline
\end{tabular}

position results fit very well with the true signal criterion. ${ }^{21}$ Consequently, although the output voltage and current of SCLNG is small, they are generated by piezoelectric $\mathrm{ZnO}$ microwire, which implies that it is possible to greatly increase the output electricity through integrating millions of piezoelectric wires. ${ }^{10,22}$

When the bar magnet moves far away from the magnetic cap on the $\mathrm{ZnO}$ microwire to close it, the microwire will be bended gradually. The final distance $r_{0}$ between the bar magnet and the magnetic cap determines the bending force applied on the $\mathrm{ZnO}$ microwire (inset of Figure 2a), which further determines the strain of $\mathrm{ZnO}$ microwire. The strain will increase with the decreasing of $r_{0}$. As a result, the piezoelectric potential between the upper side and bottom side of $\mathrm{ZnO}$ microwire will increase and the larger output voltage of SCLNG can be expected. As shown in Figure 2a, the output voltage increases from 17 to 43 $\mu \mathrm{V}$ with $r_{0}$ decreasing from 10 to $6 \mathrm{~mm}$. This relation is further plotted in Figure $2 \mathrm{~b}$, which can be understood through the following calculation.

If the bar magnet is approximately taken as a solenoid, the magnetic field on its axis line can be ${ }^{23}$

$$
B=\int_{r_{0}}^{r_{0}+L} \frac{\mu_{0} R^{2} i \mathrm{~d} r}{2\left(R^{2}+r^{2}\right)^{3 / 2}}
$$

where $B$ is the magnetic displacement, $r_{0}$ is the distance between the test point and the top surface of solenoid, $L$ is the length of the electricity solenoid, $R$ is the radius of the electricity solenoid, $i$ is the current density, and $\mu_{0}$ is the space permeability. The magnetic force acting on the magnetic cap of 

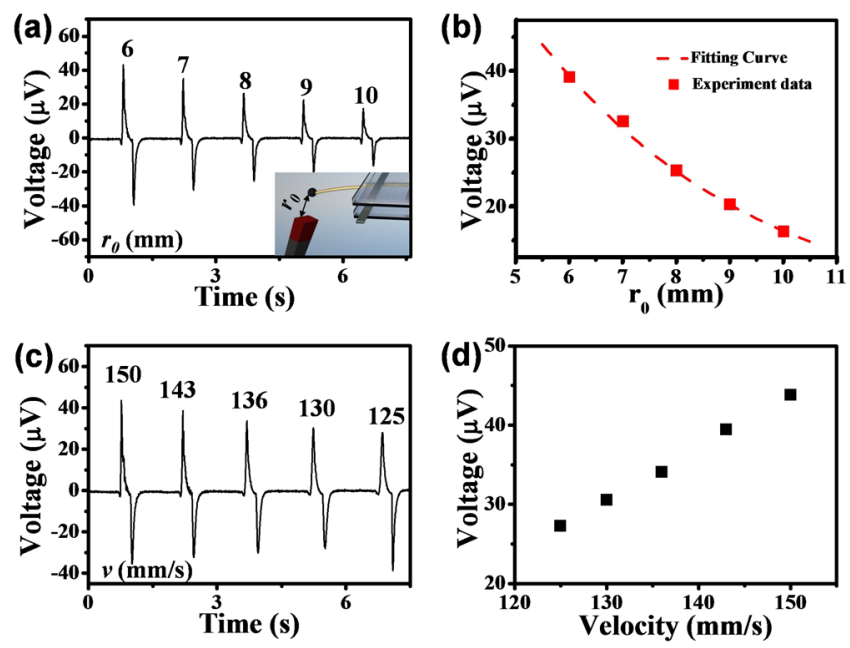

Figure 2. $(\mathrm{a}, \mathrm{b})$ The output voltage of the SCLNG as a function of the final distance $r_{0} . r_{0}$ is defined as the distance between the magnet and the magnetic cap on $\mathrm{ZnO}$ microwire as shown in the inset of panel a. The dash curve in panel $b$ is the theoretical fitting curve. (c) The output voltage of SCLNG at different movement velocity of magnet. (d) The plot of output voltage with the movement velocity of the magnet.

SCLNG is proportional to the magnetic field gradient. The magnetic field gradient along the axis line is

$$
\begin{aligned}
\nabla B & =C_{1}\left(\frac{1}{\sqrt{\left(r_{0}+L\right)^{2}+C_{2}}}-\frac{\left(r_{0}+L\right)^{2}}{\left(\left(r_{0}+L\right)^{2}+C_{2}\right)^{3 / 2}}\right. \\
+ & \left.\frac{r_{0}^{2}}{\left(r_{0}^{2}+C_{2}\right)^{3 / 2}}-\frac{1}{\sqrt{r_{0}^{2}+C_{2}}}\right), \quad C_{1}=\frac{i \mu_{0}}{2}, \\
C_{2} & =R^{2}
\end{aligned}
$$

Then regard the magnetization $M$ of the magnetic cap as a constant, the magnetic force will be proportional to the magnetic field gradient

$$
\begin{aligned}
F \propto & \nabla B \\
= & C_{1}\left(\frac{1}{\sqrt{\left(r_{0}+L\right)^{2}+C_{2}}}-\frac{\left(r_{0}+L\right)^{2}}{\left(\left(r_{0}+L\right)^{2}+C_{2}\right)^{3 / 2}}\right. \\
& \left.+\frac{r_{0}^{2}}{\left(r_{0}^{2}+C_{2}\right)^{3 / 2}}-\frac{1}{\sqrt{r_{0}^{2}+C_{2}}}\right)
\end{aligned}
$$

As described in ref 24, the maximum potential at the surface of the NW at the tensile ( $\mathrm{T}$ ) side and the compressive (C) side, respectively, being

$$
\varphi_{\max }^{(\mathrm{T}, \mathrm{C})}= \pm \frac{1}{\pi\left(\kappa_{0}+\kappa_{\perp}\right)} \frac{F}{E}\left[e_{33}-2(1+\nu) e_{15}-2 \nu e_{31}\right] \frac{1}{a}
$$

where $\kappa$ is the dielectric constant, $e$ is the linear piezoelectric coefficient, $E$ is the Young's modulus, $\nu$ is the Poisson ratio, and $a$ is the radius of the $\mathrm{ZnO}$ microwire. Through eqs $2-4$ we can get

$$
\begin{aligned}
\varphi_{\max }^{(\mathrm{T}, \mathrm{C})} \propto & \pm C_{3} C_{1}\left(\frac{1}{\sqrt{\left(r_{0}+L\right)^{2}+C_{2}}}\right. \\
& -\frac{\left(r_{0}+L\right)^{2}}{\left(\left(r_{0}+L\right)^{2}+C_{2}\right)^{3 / 2}}+\frac{r_{0}^{2}}{\left(r_{0}^{2}+C_{2}\right)^{3 / 2}} \\
& \left.-\frac{1}{\sqrt{r_{0}^{2}+C_{2}}}\right) \\
C_{3}= & \frac{1}{\pi\left(\kappa_{0}+\kappa_{\perp}\right)} \frac{1}{E}\left[e_{33}-2(1+\nu) e_{15}-2 \nu e_{31}\right] \frac{1}{a}
\end{aligned}
$$

By calculating eq 5 with proper parameters, the fitting curve of output voltage and the distance $r_{0}$ agrees well with the experimental data shown in Figure $2 \mathrm{~b}$. This curve reflects the output voltage of SCLNG as a function of the distance $r_{0}$.

When the distance $r_{0}$ is kept at $6 \mathrm{~mm}$, the output voltage of SCLNG increases with the average moving velocity of the magnet, which is shown in Figure $2 \mathrm{c}$,d. If $r_{0}$ is a constant, the maximum strain in the $\mathrm{ZnO}$ microwire will be a constant. When the velocity increase, less time will be spent to bend the $\mathrm{ZnO}$ microwire to the maximum strain state. The same amount of electrons in external circuit will flow more quickly from the negative potential side of $\mathrm{ZnO}$ microwire to its positive side, which leads to higher output voltage. When the $\mathrm{ZnO}$ microwire recovers from the bending state to freedom, the same variation trend can be obsered. As a result, the output voltage of SCLNG increases with the average velocity of the magnet.

Integrated Contactless Nanogenerator (ICLNG) Based on PZT Nanowire Array. The bulk piezoelectric coefficient of $\mathrm{PZT}$ is $500-600 \mathrm{pC} / \mathrm{N}^{25-28}$ which is about 50 times of the value $11.67 \mathrm{pC} / \mathrm{N}$ of bulk $\mathrm{ZnO}^{29}$ So higher piezoelectric potential can be expected for the PZT nanowire comparing with that of the $\mathrm{ZnO}$ nanowire when their strain and size are same. At the same time, nanowires have good mechanical performance, such as flexibility, fatigue durability, and so forth. ${ }^{30}$ So PZT nanowires should be a good candidate for the high output ICLNG. Here, we use the electrospinning method to make well aligned PZT nanowires as shown in Figure 3a. The inset of this figure reveals that these nanowires have perovskite crystal structure. Figure $3 \mathrm{~b}$ shows the structure of ICLNG, which can be fabricated through the following steps. First, a magnetic layer composed of $\mathrm{Fe}_{3} \mathrm{O}_{4}$ and PDMS is acted as a driven layer under the variance of magnetic field. Its top is covered with another insulation layer consisting of the mixture of quartz power and polydimethylsiloxane (PDMS). Then the well-aligned PZT nanowires are put onto this insulation layer. Photolithography and magnetron sputtering are used in sequence to fabricate $\mathrm{Ag}$ electrodes connecting PZT NWs. After that, PDMS is used to package the ICLNG. The inset is the picture of a packaged ICLNG. Finally, the ICLNG is polarized by applying an electric field of $4 \mathrm{~V} / \mu \mathrm{m}$ at $130{ }^{\circ} \mathrm{C}$ for about $10 \mathrm{~min}$.

Figure $3 \mathrm{c}$ shows the working mode of ICLNG. At the initial state, the thick substrate film is bended by fixing its two ends onto a glass slide. At this moment, PZT nanowires are stretched. When NdFeB magnet approachs the ICLNG, the whole device will be adsorbed flatly onto the surface of the glass slide. Because the two ends of ICLNG are fixed, PZT nanowires are compressed completely with its polymer substrate together. During this process, PZT nanowires are stretched, released, and then compressed successively. The 


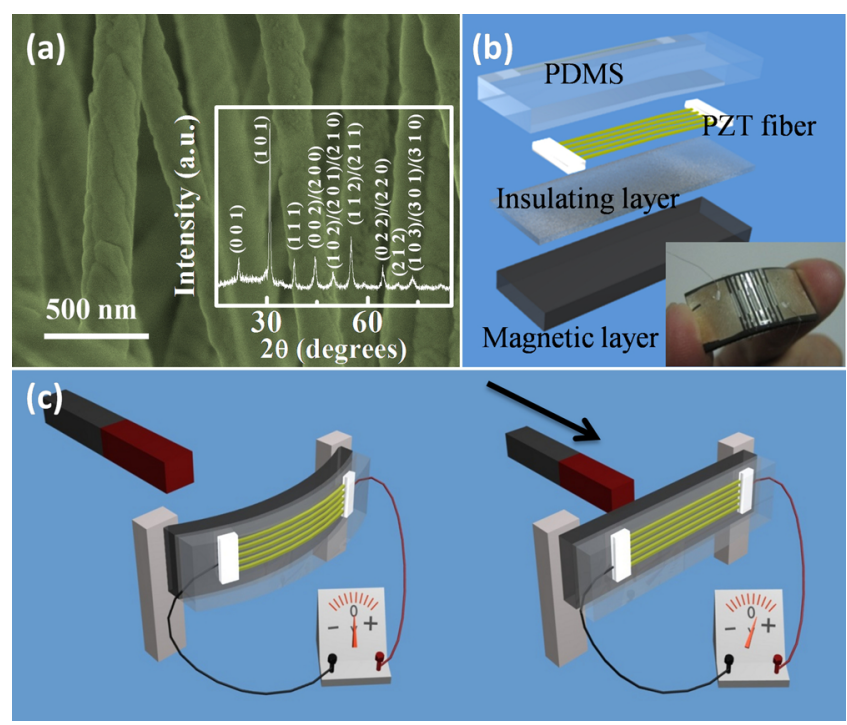

Figure 3. Structure and working mode of the ICLNG. (a) SEM image of the parallel-aligned PZT nanowire array. The inset is its X-ray diffraction spectrum. (b) Schematic structure of the ICLNG. PZT nanowires are put onto an insulating layer covered magnetic flexible composite, connected with two electrodes, and then packaged with PDMS. The inset is the photograph of a real ICLNG. (c) Working mode of the ICLNG. The magnetic layer converts the movement energy of magnet into the strain change of PZT nanowires. The piezoelectric potential between two ends of nanowires drives electrons flowing. Therefore, ICLNG generates electricity.

electrons in external circuit continuously flow from one end of the nanowires to the other end through the external load and accumulated at the interface between the metal electrode and PZT nanowires. As the magnet departs from the ICLNG, PZT nanowires release from the compressing state, and then become stretched under the influence of the polymer substrate film. This process will makes the accumulated electrons flow back in external circuit. As a result, the electrons will flow back and forth with the magnet approaching and departing from the ICLNG. The nanogenerator will generate alternating current as shown in Figure 4.
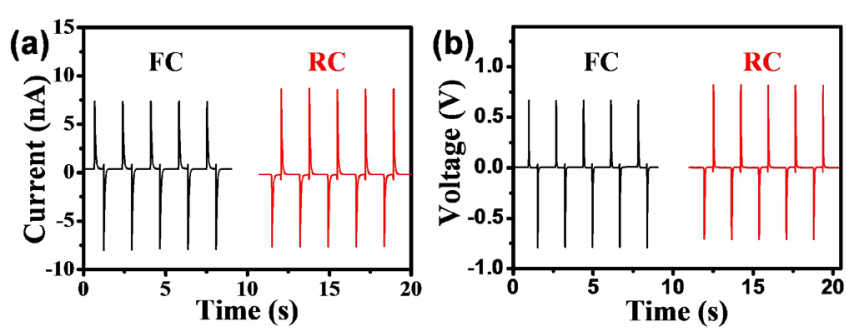

Figure 4. The output current (a) and voltage (b) of the ICLNG. FC means forward connection, and RC means reverse connection.

Figure $4 \mathrm{a}, \mathrm{b}$ respectively shows the output current and voltage of an ICLNG made of a $2 \mathrm{~mm} \times 0.5 \mathrm{~mm}$ PZT nanowire array film with the thickness of $5 \mu \mathrm{m}$. The output current is about several nanoamperes, and the output voltage is slightly less than $1 \mathrm{~V}$. As shown in Figure 4, when we reversely connected the measurement system with the ICLNG, the output current and voltage reversed their signs, which means that the output current and voltage are true signals.
To further increase the output electricity of ICLNG, five pieces of PZT nanowire array film were connected in series on the polymer substrate. The total area of active PZT films is about $17.5 \mathrm{~mm}^{2}$, and their thickness is still $5 \mu \mathrm{m}$. So the total volume of PZT nanowire is about $8.8 \times 10^{-5} \mathrm{~cm}^{3}$. Figure $5 \mathrm{a}, \mathrm{b}$
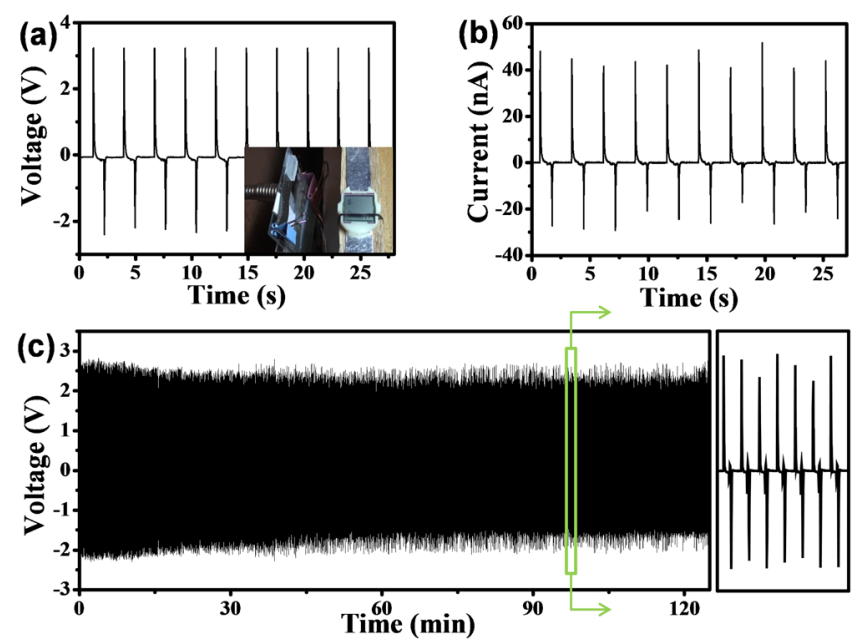

Figure 5. The output voltage (a) and current (b) of the ICLNG composed of five pieces of PZT nanowire array connected in series. The inset is a photograph of the LCD screen lit by the ICLNG. (c) The electrical output of a ICLNG working at a frequency of 34 cycles per minute. The more than $2 \mathrm{~h}$ of continuously working of the ICLNG demonstrates its stability and robustness.

shows the performance of this device. The maximum output voltage reached $3.2 \mathrm{~V}$, and the corresponding output current was about $50 \mathrm{nA}$. Considering the load resistance of $100 \mathrm{M} \Omega$, the maximum output power density of this integrated nanogenerator is about $170 \mu \mathrm{W} / \mathrm{cm}^{3}$. The output electricity of ICLNG is high enough to light a commercial LCD. An LCD screen taken from a personal electronic watch is connected to the ICLNG. As shown in the inset of Figure 5a and Supporting Information video, when the magnet approachs the ICLNG, a character appears on the LCD screen and then disappears. When the magnet leaves the ICLNG, the character appears again. In other words, during one cycle movement of magnet, the LCD character can be lit twice, which is due to the presence of two output voltage peaks in this process.

Because the ICLNG is completely packaged, it has very high robustness. Figure $5 c$ shows the stability of the ICLNG driven at a frequency of 34 cycles per minute. In this figure, the right curve is an enlarged view of the signals surrounded by the rectangular box in the left curve. Even after continuously working more than $2 \mathrm{~h}$, no obvious damage of ICLNG can be seen. More importantly, the output signal is still as large as that at the beginning, which implies that no decay in performance appears for ICLNG after working so long time.

In conclusion, two different kinds of noncontact magnetic force driven nanogenerators have been developed. One uses the piezopotential drop along the radial direction of nanowire to generate electricity, and the other uses the piezopotential drop along its axial direction to generate electricity. Both of them can work without contacting with the mechanical movement source. Using a single $\mathrm{ZnO}$ microwire, we first proved the feasibility of the noncontact magnetic force driven generator. Subsequently, the electrospinning PZT nanowire array is used to make an ICLNG working in noncontact mode. This kind of 
nanogenerator has given an output voltage of $3.2 \mathrm{~V}$ and output current of $50 \mathrm{nA}$. The maximum output power density of this device was $170 \mu \mathrm{W} / \mathrm{cm}^{3}$. This nanogenerator was successfully demonstrated to light an LCD screen. The magnetic driven nanogenerator paves the way to the noncontact mechanical energy harvesting and its possibility for magnetic sensing using nanogenerators.

\section{ASSOCIATED CONTENT}

\section{S Supporting Information}

Additional figures about the output current and voltage of two SCLNGs connected in series and in parallel. Additional video showing the LCD screen lit by the ICLNG. This material is available free of charge via the Internet at http://pubs.acs.org.

\section{AUTHOR INFORMATION}

\section{Corresponding Author}

*E-mail: (Y.Q.) qinyong@lzu.edu.cn; (Z.L.W.) zhong.wang@ mse.gatech.edu.

\section{Notes}

The authors declare no competing financial interest.

\section{ACKNOWLEDGMENTS}

We gratefully acknowledge the financial support from NSFC (NO. 50972053, 11034004), Fok Ying Tung education foundation (131044), Ph.D. Programs Foundation of Ministry of Education of China (No. 20090211110026), the Fundamental Research Funds for the Central Universities (No. lzujbky-2010-k01), NCET (No. NCET-08).

\section{REFERENCES}

(1) Dresselhaus, M. S.; Chen, G.; Tang, M. Y.; Yang, R.; Lee, H.; Wang, D.; Ren, Z.; Fleurial, J. P.; Gogna, P. Adv. Mater. 2007, 19 (8), 1043-1053.

(2) Hagerty, J. A.; Helmbrecht, F. B.; McCalpin, W. H.; Zane, R.; Popovic, Z. B. IEEE Trans. Microwave Theory Tech. 2004, 52 (3), $1014-1024$

(3) McSpadden, J. O.; Fan, L.; Chang, K. IEEE Trans. Microwave Theory Tech. 1998, 46 (12), 2053-2060.

(4) Tian, B.; Zheng, X.; Kempa, T. J.; Fang, Y.; Yu, N.; Yu, G.; Huang, J.; Lieber, C. M. Nature 2007, 449 (7164), 885.

(5) Wang, Z. L.; Song, J. Science 2006, 312 (5771), 242.

(6) Weintraub, B.; Wei, Y.; Wang, Z. L. Angew. Chem., Int. Ed. 2009, 48 (47), 8981-5.

(7) Yu, C.; Shi, L.; Yao, Z.; Li, D.; Majumdar, A. Nano Lett. 2005, 5 (9), 1842-1846.

(8) Wang, Z. L. Nano Today 2010, 5 (6), 512-514.

(9) Wang, Z. L. Nanogenerators for Self-powered Devices and Systems; Georgia Institute of Technology: Atlanta, GA, 2011.

(10) Xu, S.; Qin, Y.; Xu, C.; Wei, Y.; Yang, R.; Wang, Z. L. Nat. Nanotechnol. 2010, 5 (5), 366-373.

(11) Yang, R.; Qin, Y.; Li, C.; Zhu, G.; Wang, Z. L. Nano Lett. 2009, 9 (3), 1201-1205.

(12) Wang, X. D.; Zhou, J.; Song, J. H.; Liu, J.; Xu, N. S.; Wang, Z. L. Nano Lett. 2006, 6 (12), 2768-2772.

(13) Wang, X.; Song, J.; Liu, J.; Wang, Z. L. Science 2007, 316 (5821), $102-5$.

(14) Qin, Y.; Wang, X.; Wang, Z. L. Nature 2008, 451 (7180), 809813.

(15) Hu, Y.; Lin, L.; Zhang, Y.; Wang, Z. L. Adv. Mater. 2012, 24 (1), $110-4$.

(16) Hu, Y. F.; Zhang, Y.; Xu, C.; Zhu, G. A.; Wang, Z. L. Nano Lett. 2010, 10 (12), 5025-5031.

(17) Zhu, G. A.; Yang, R. S.; Wang, S. H.; Wang, Z. L. Nano Lett. 2010, 10 (8), 3151-3155.
(18) Wang, X.; Liu, J.; Song, J.; Wang, Z. L. Nano Lett. 2007, 7 (8), $2475-2479$.

(19) Zhou, J.; Fei, P.; Gao, Y.; Gu, Y.; Liu, J.; Bao, G.; Wang, Z. L. Nano Lett. 2008, 8 (9), 2725-2730.

(20) Yang, R. S.; Qin, Y.; Dai, L. M.; Wang, Z. L. Nat. Nanotechnol. 2009, 4 (1), 34-39.

(21) Yang, R. S.; Qin, Y.; Li, C.; Dai, L. M.; Wang, Z. L. Appl. Phys. Lett. 2009, 94, 2.

(22) Wang, Z. L.; Yang, R. S.; Zhou, J.; Qin, Y.; Xu, C.; Hu, Y. F.; Xu, S. Mater. Sci. Eng., R. 2010, 70 (3-6), 320-329.

(23) Zhao, K. H.; Chen, X. M. Electromagnetism; Higher Education Press: Beijing, 2006.

(24) Gao, Y.; Wang, Z. L. Nano Lett. 2007, 7 (8), 2499-2505.

(25) Ren, X. Nat. Mater. 2004, 3 (2), 91-94.

(26) Saito, Y.; Takao, H.; Tani, T.; Nonoyama, T.; Takatori, K.; Homma, T.; Nagaya, T.; Nakamura, M. Nature 2004, 432 (7013), 8487.

(27) Shrout, T. R.; Zhang, S. J. J. Electroceram. 2007, 19 (1), 113126.

(28) Takenaka, T.; Nagata, H. J. Eur. Ceram. Soc. 2005, 25 (12), 2693-2700.

(29) http://www.efunda.com/materials/piezo/material_data/ matdata_output.cfm?Material_ID=ZnO (accessed September, 2005).

(30) Han, X.; Zheng, K.; Zhang, Y. F.; Zhang, X.; Zhang, Z.; Wang, Z. L. Adv. Mater. 2007, 19 (16), 2112-2118. 